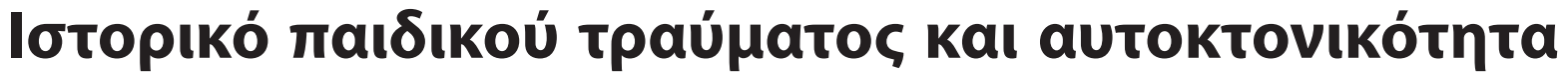

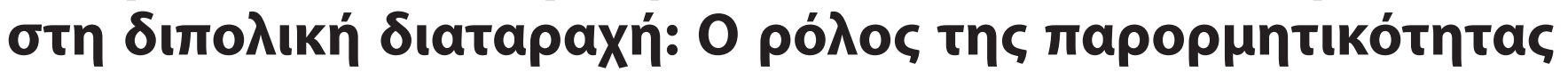

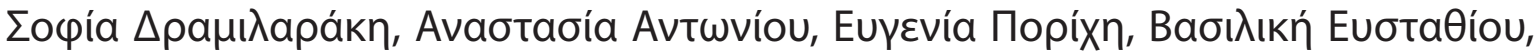

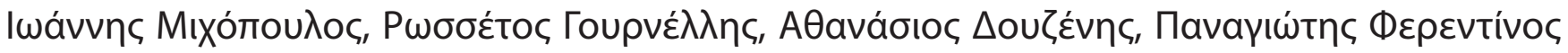

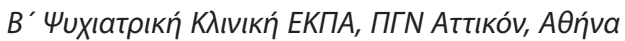

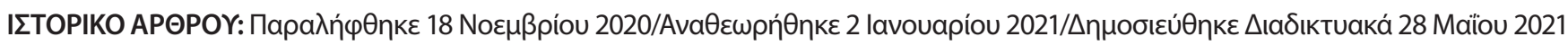

\begin{abstract}
ПЕРІЛНЧН

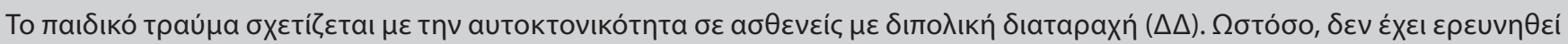

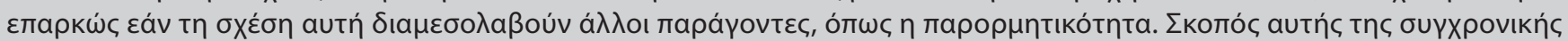

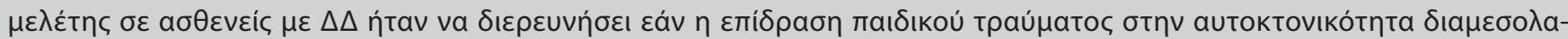

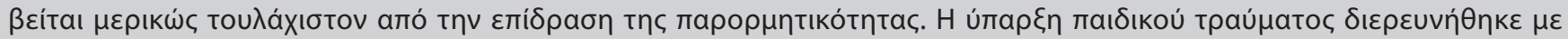

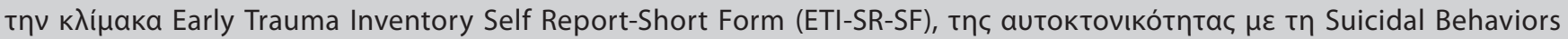

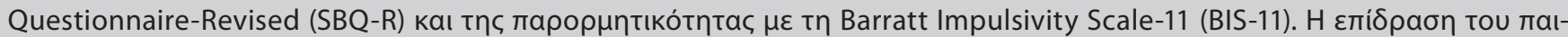

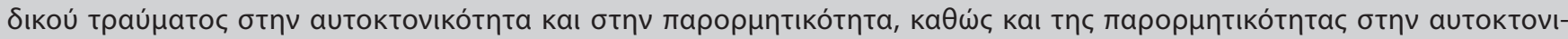

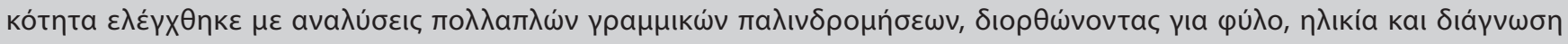

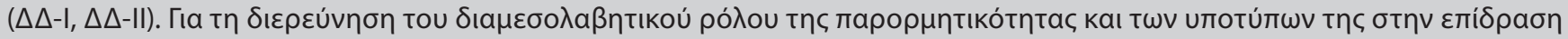

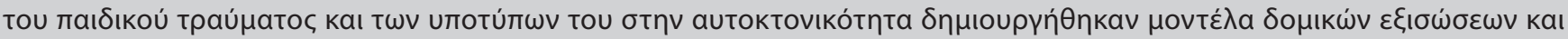

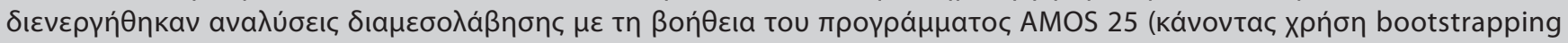

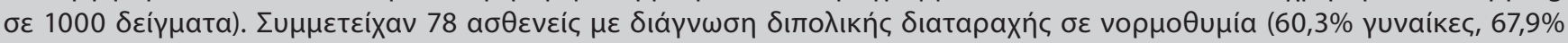

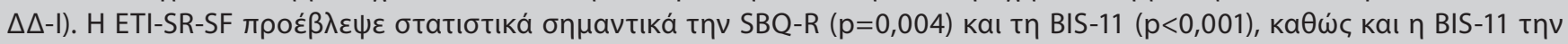

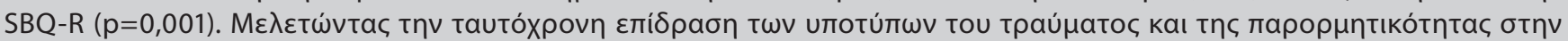

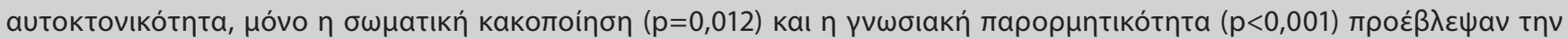

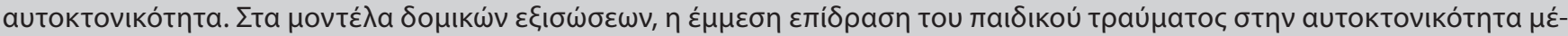

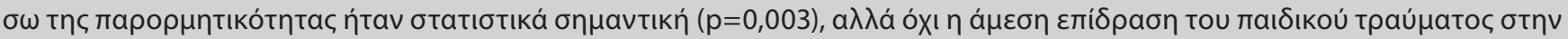

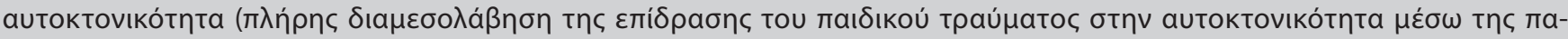

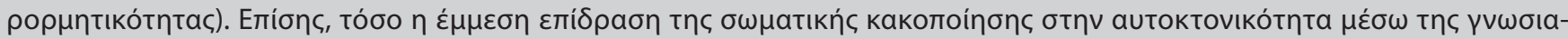

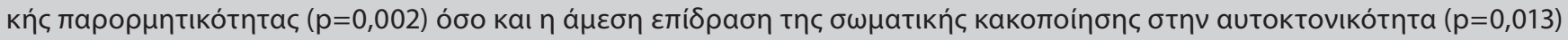

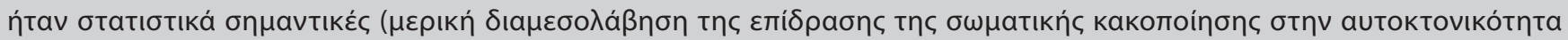

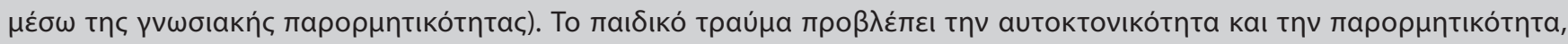

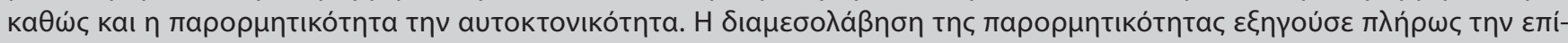

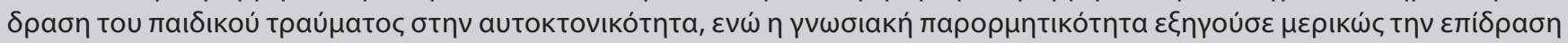

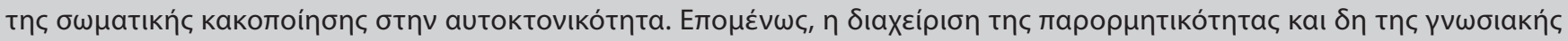

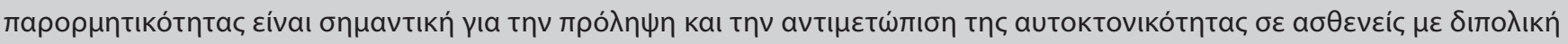

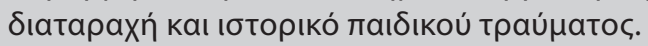

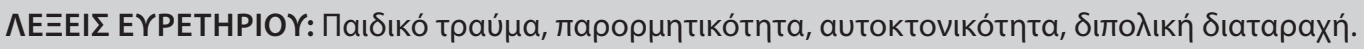




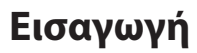

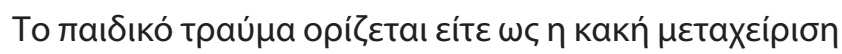

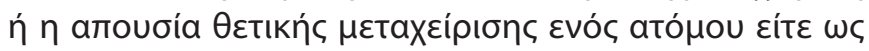

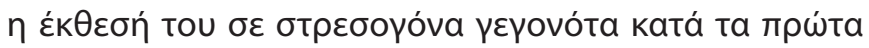

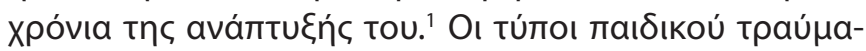

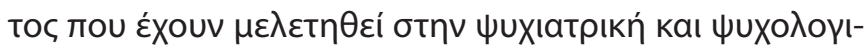

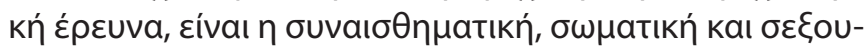

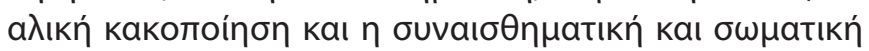

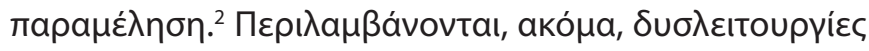

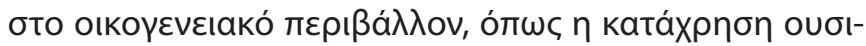

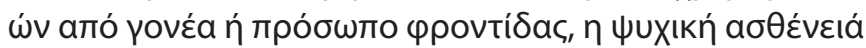

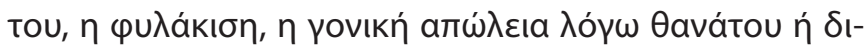

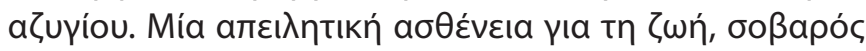

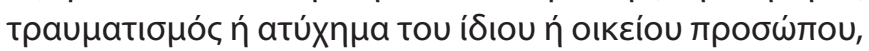

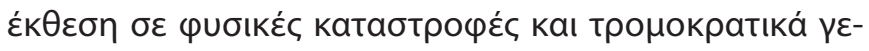

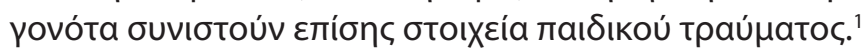

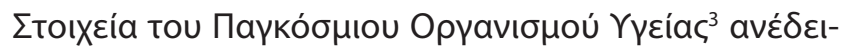

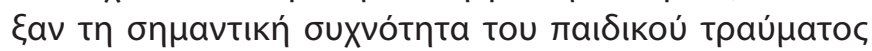

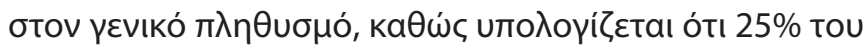

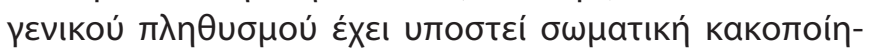

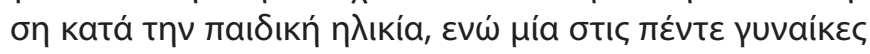

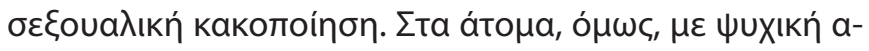

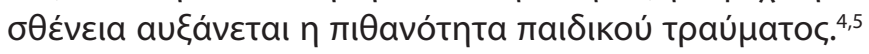

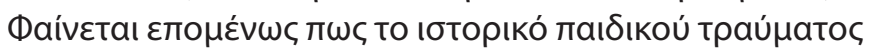

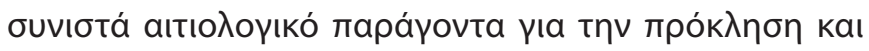

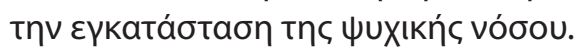

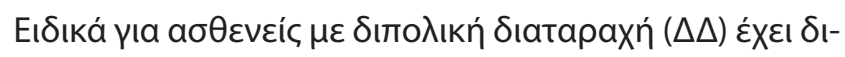

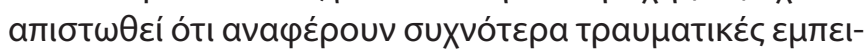

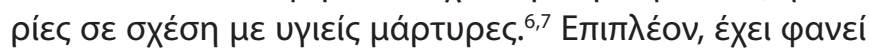

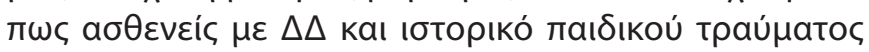

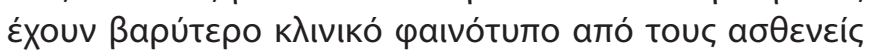
X

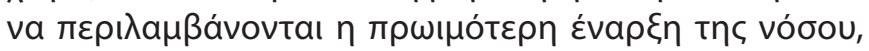

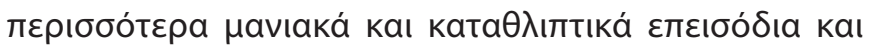

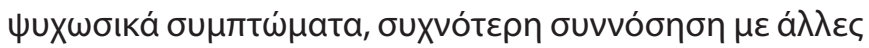

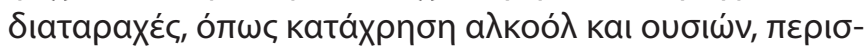

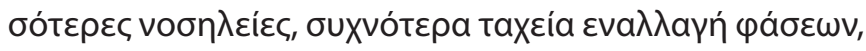

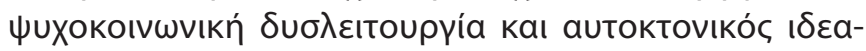

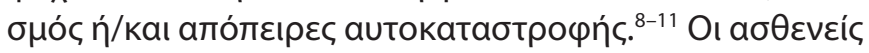

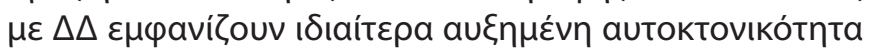

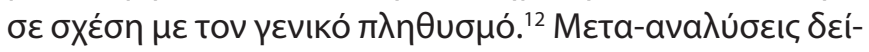

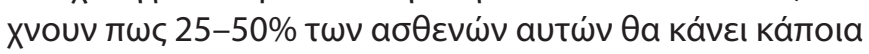

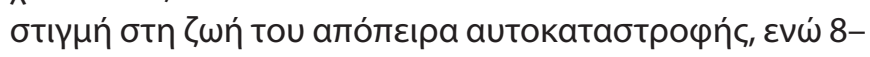

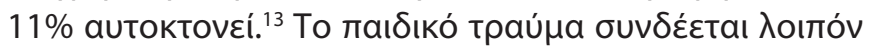

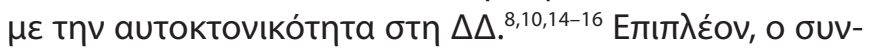

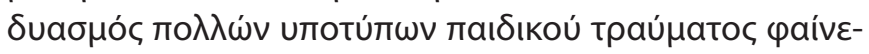

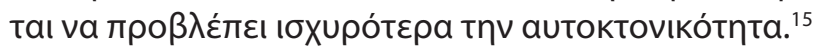

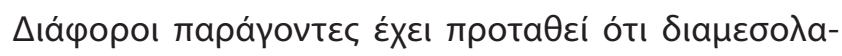

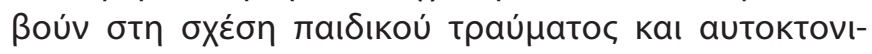

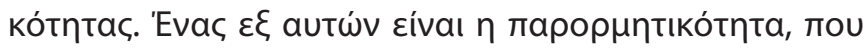

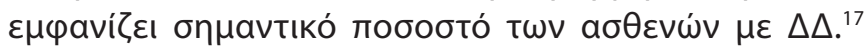

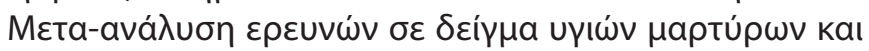

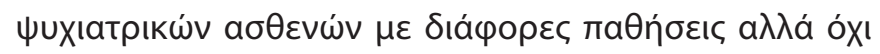

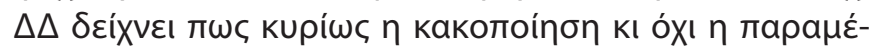

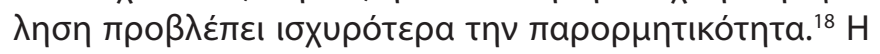

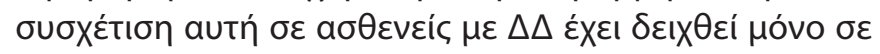

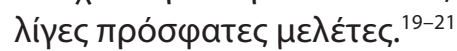

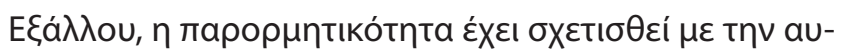

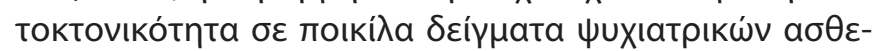

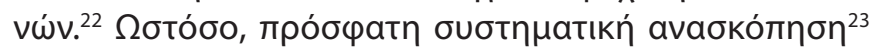

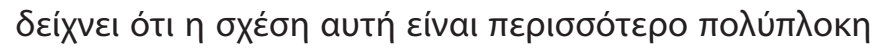

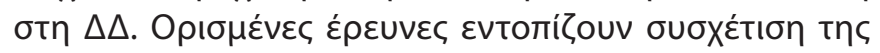

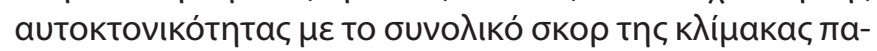

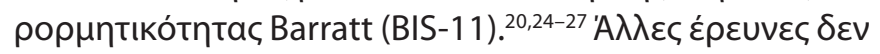

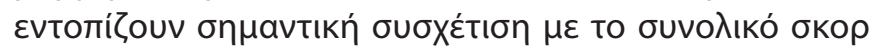

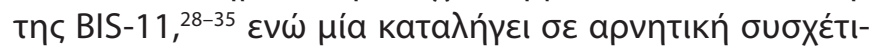

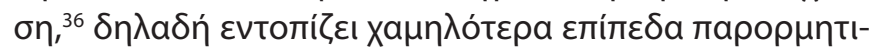

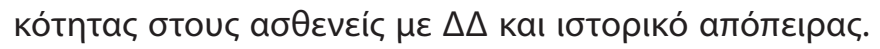

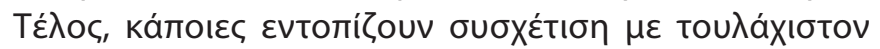

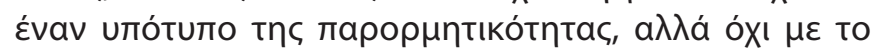

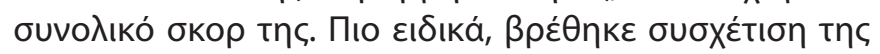

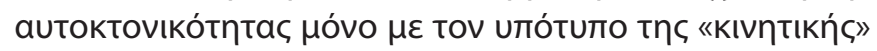

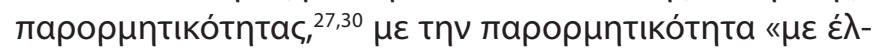

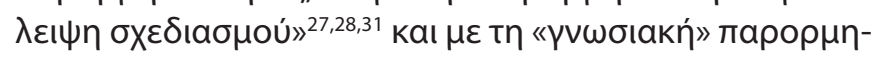
тIKótnтa. ${ }^{28}$

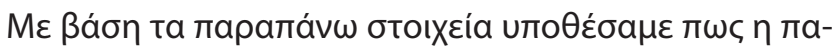

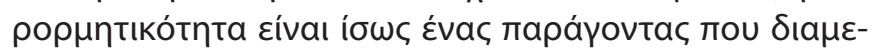

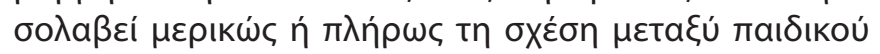

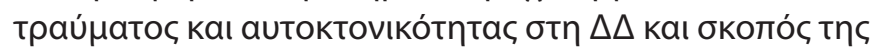

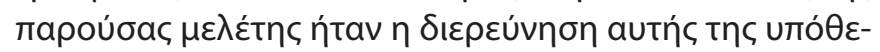

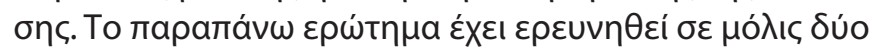

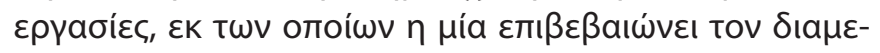

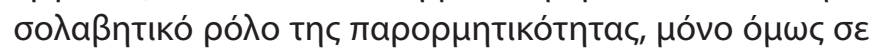

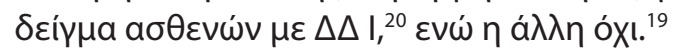

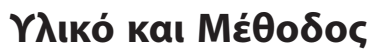

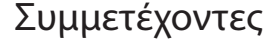

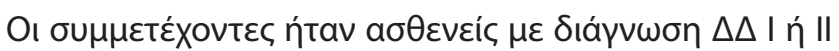

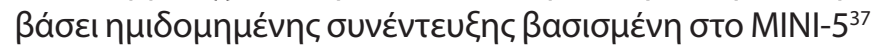

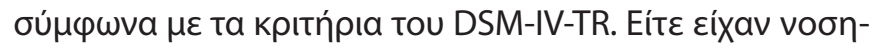

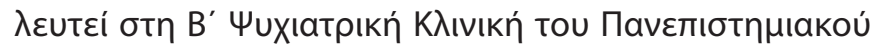

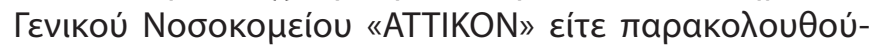

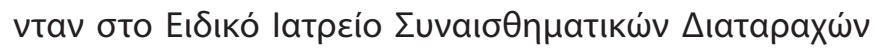

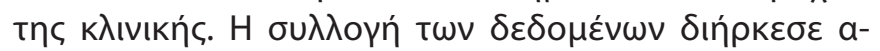

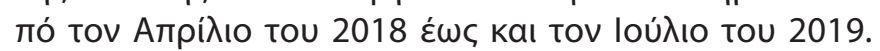

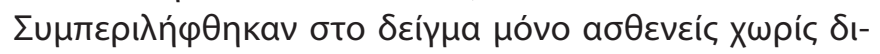

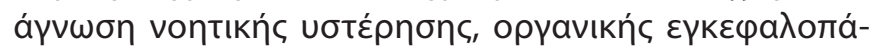

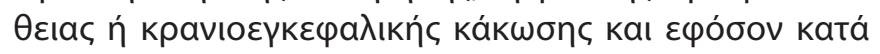

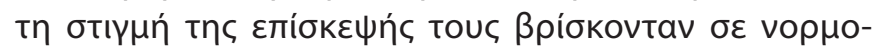

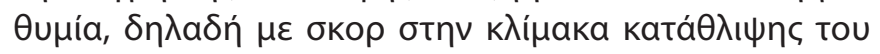

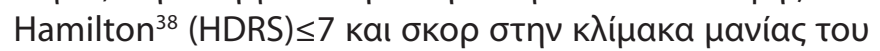

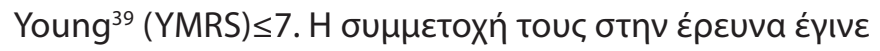

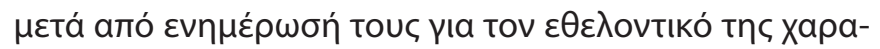

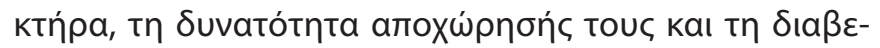




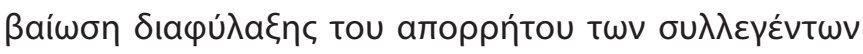

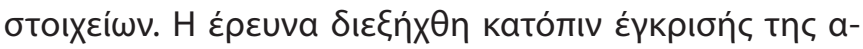

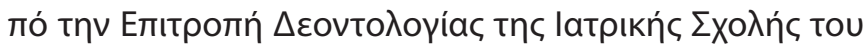

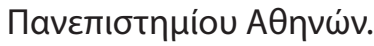

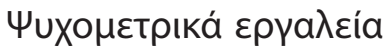

\section{Early Trauma Inventory-Self Report-Short Form (ETI-SR-SF)}

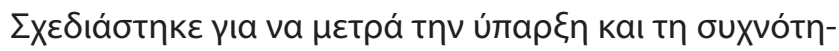

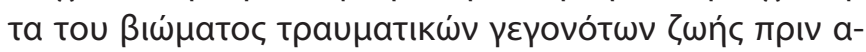

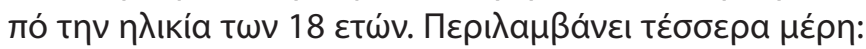

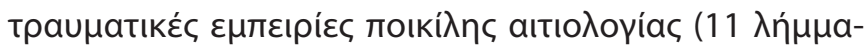

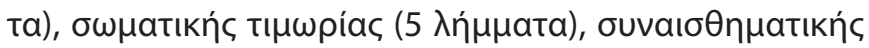

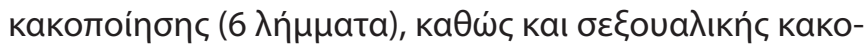

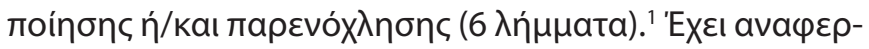

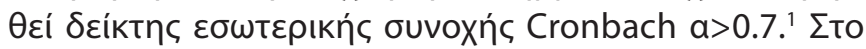

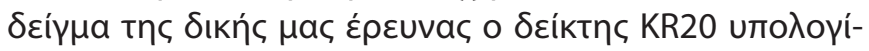

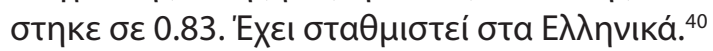

\section{The Suicide Behaviors Questionnaire-Revised (SBQ-R)}

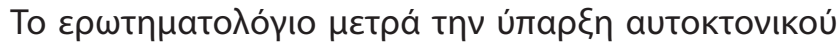

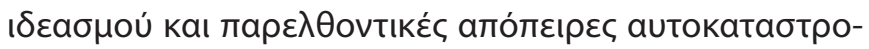

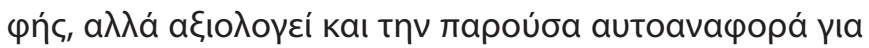

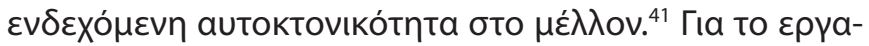

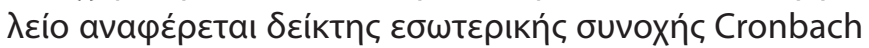

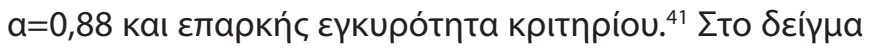

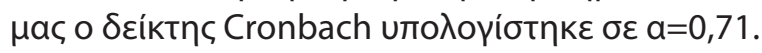

\section{Barratt Impulsiveness Scale (BIS-11)}

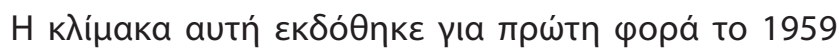

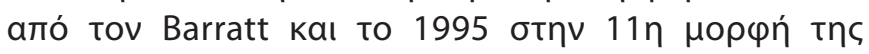

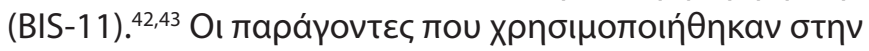

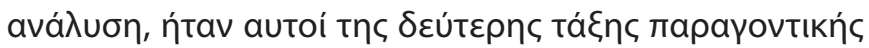

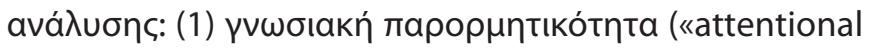

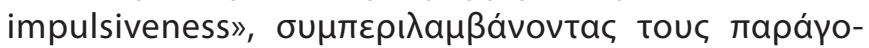

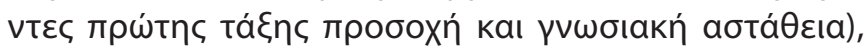

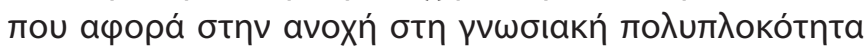

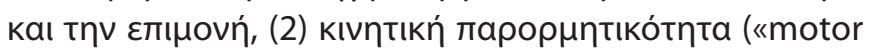

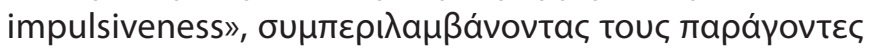

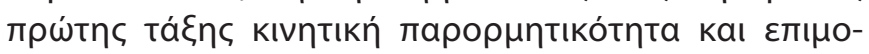

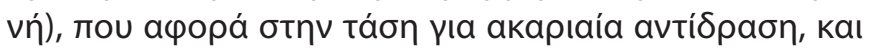

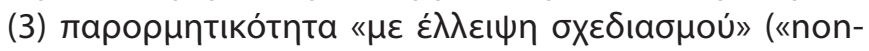

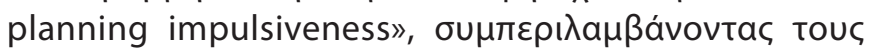

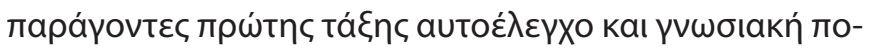

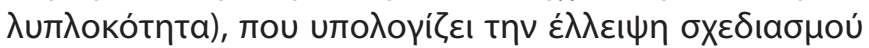

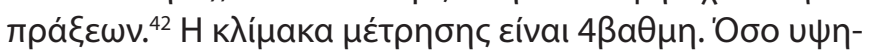

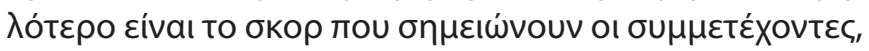

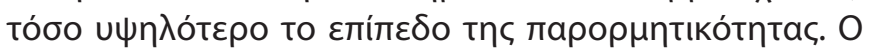

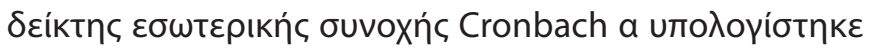

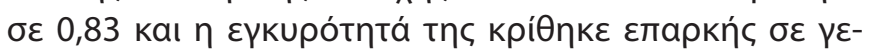

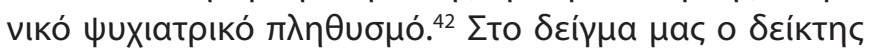

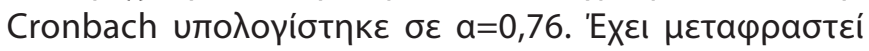

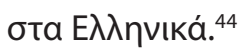

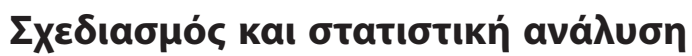

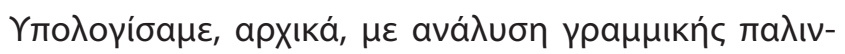

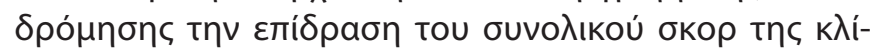

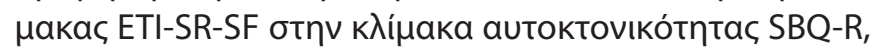

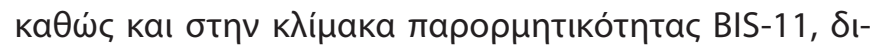

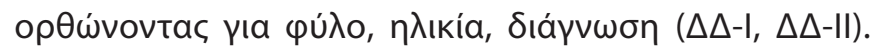

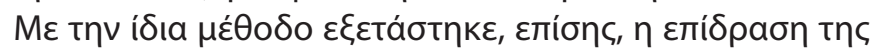

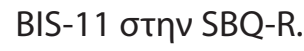

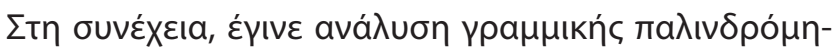

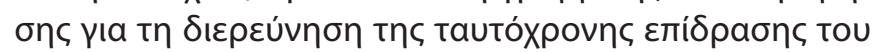

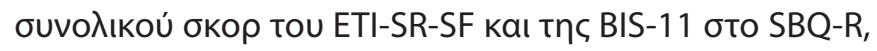

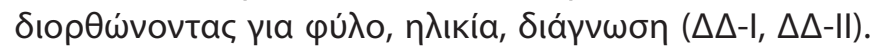

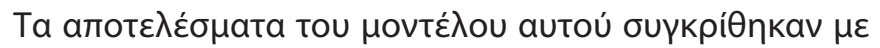

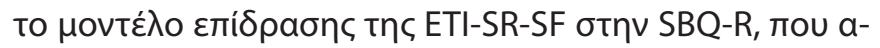

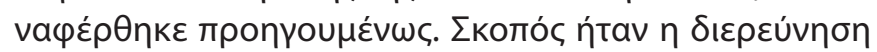

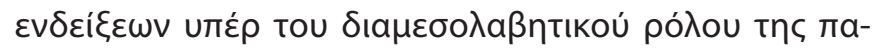

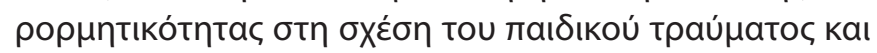
тทৎ autokToviкótntac.

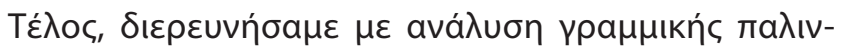

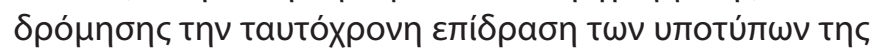

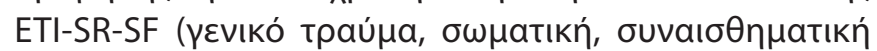

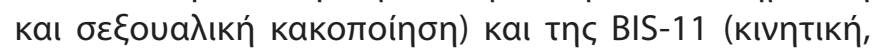

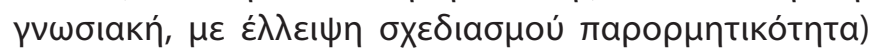

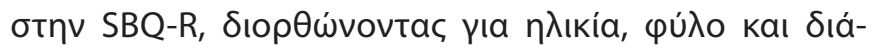

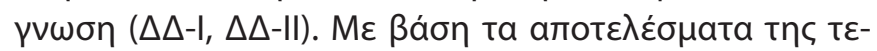

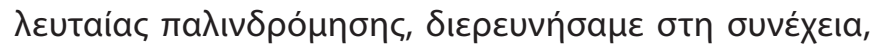

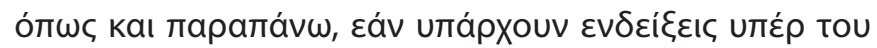

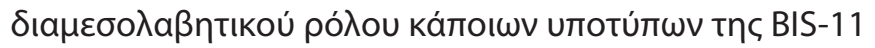

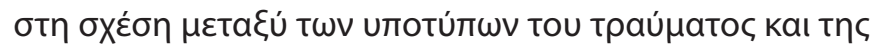
SBQ-R.

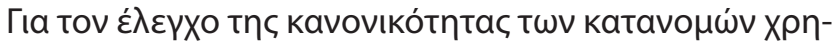

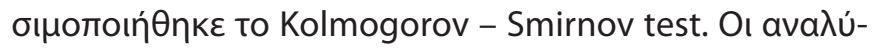

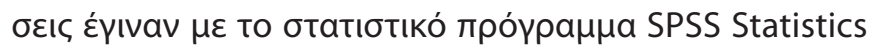

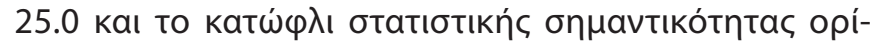

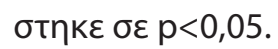

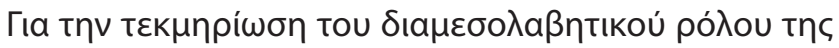

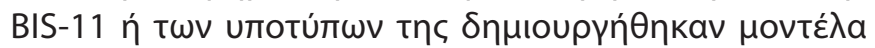

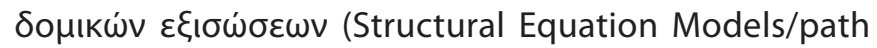

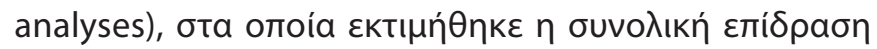

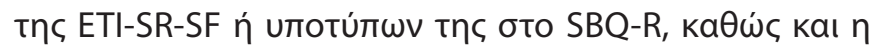

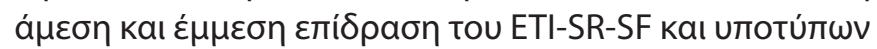

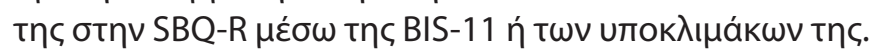

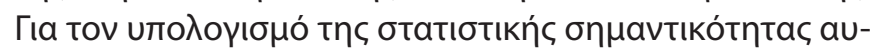

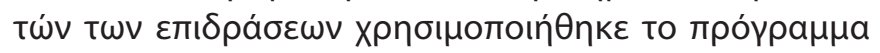

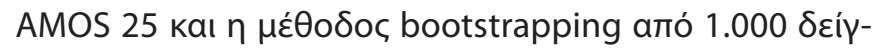
Mata.

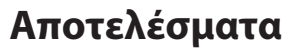

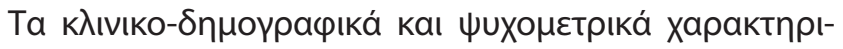

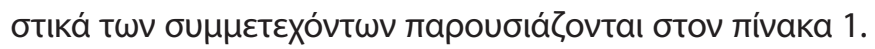

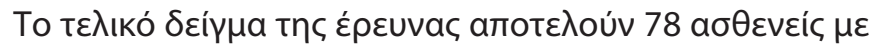




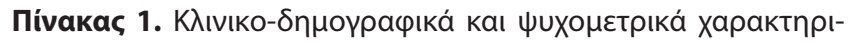

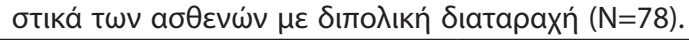

\begin{tabular}{|c|c|}
\hline 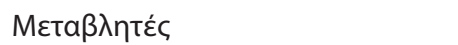 & 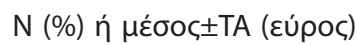 \\
\hline 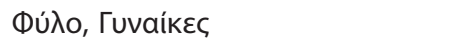 & $47(60,3)$ \\
\hline 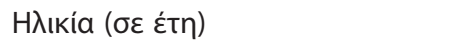 & $47 \pm 11,27(23-78)$ \\
\hline 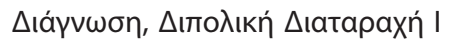 & $53(67,9)$ \\
\hline \multicolumn{2}{|l|}{ 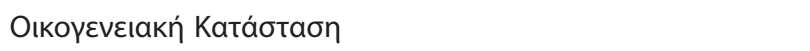 } \\
\hline 'Еүүанос-п & $25(32,1)$ \\
\hline 'Аүанос-п & $25(32,1)$ \\
\hline$\Delta \mathrm{la \zeta \varepsilon uү \mu \varepsilon ́vo \varsigma - \eta ,} \mathrm{Xń \rho o \varsigma -a}$ & $28(35,9)$ \\
\hline \multicolumn{2}{|l|}{ 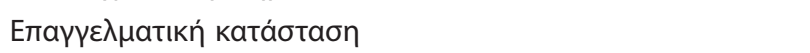 } \\
\hline 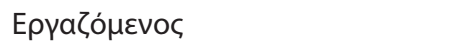 & $33(42,3)$ \\
\hline 'Aveрүo૬ & $31(39,7)$ \\
\hline ¿uvtakııúxoc & $14(17,9)$ \\
\hline 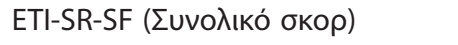 & $0,28 \pm 0,18(0-0,74)$ \\
\hline 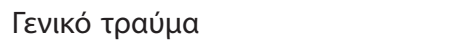 & $0,24 \pm 0,18(0-0,73)$ \\
\hline 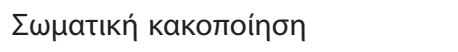 & $0,41 \pm 0,28(0-1)$ \\
\hline 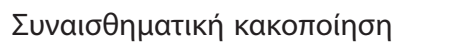 & $0,48 \pm 0,38(0-1)$ \\
\hline 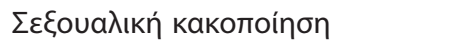 & $0,10 \pm 0,17(0-0,83)$ \\
\hline 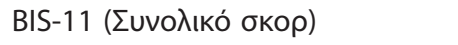 & $2,14 \pm 0,33(1,33-2,87)$ \\
\hline 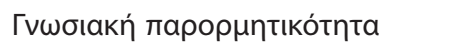 & $2,03 \pm 0,50(1,25-3,25)$ \\
\hline 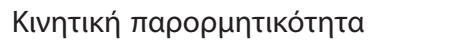 & $1,97 \pm 0,35(1,36-2,82)$ \\
\hline 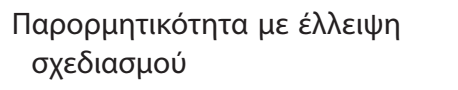 & $2,39 \pm 0,42(1,36-3,18)$ \\
\hline SBQ-R (Аитоктоvıко́тпта) & $6,94 \pm 3,78(3-17)$ \\
\hline
\end{tabular}

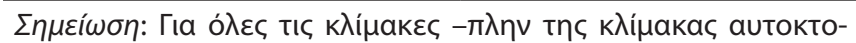

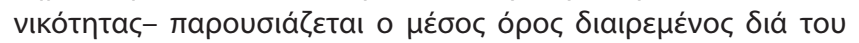

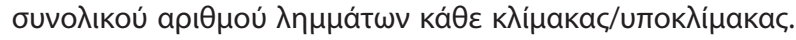

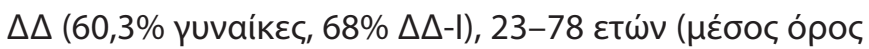

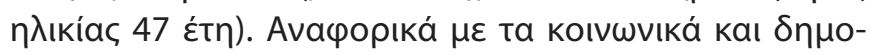

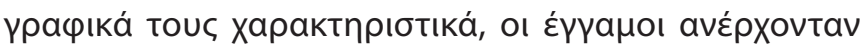

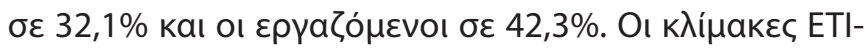

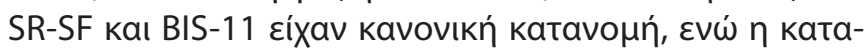

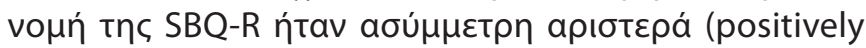

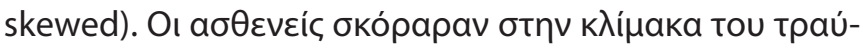

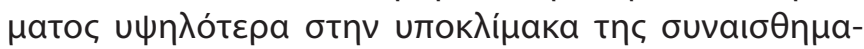

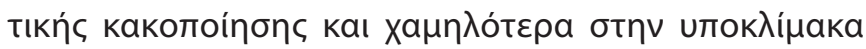

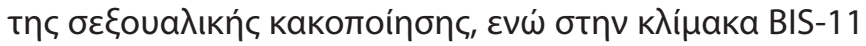

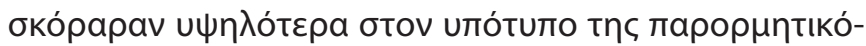

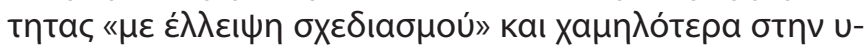

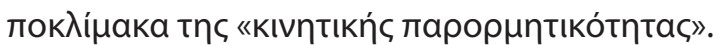

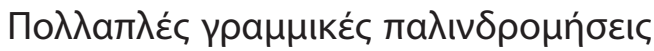

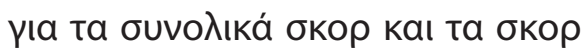

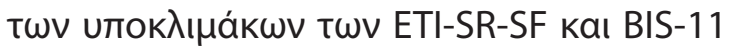

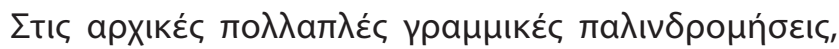

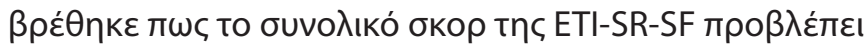

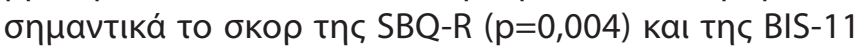

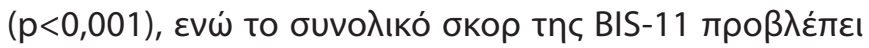

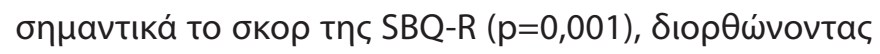

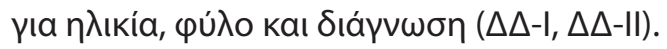

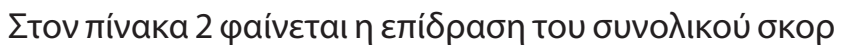

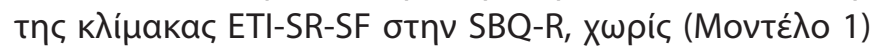

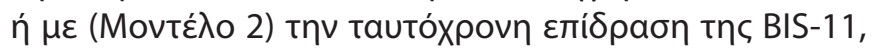

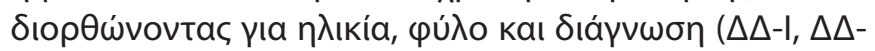

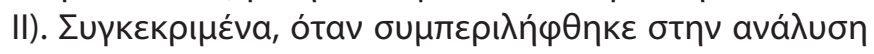

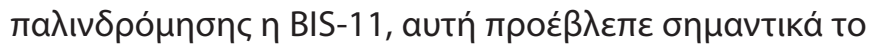

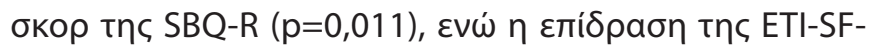

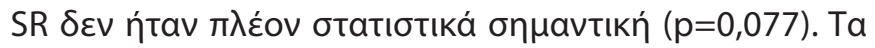

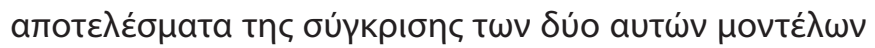

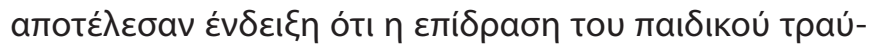

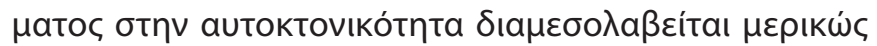

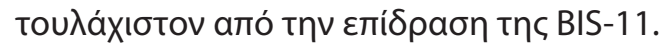

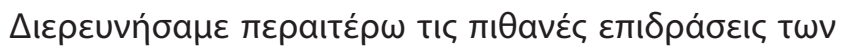

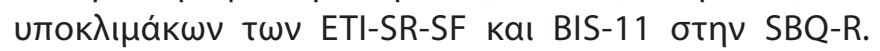

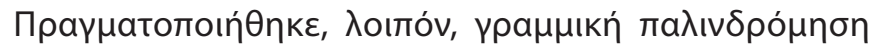

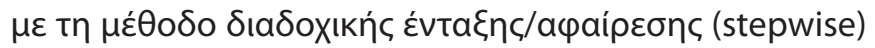

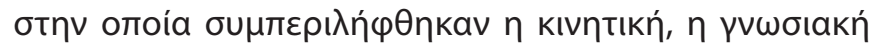

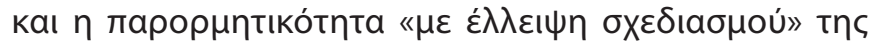

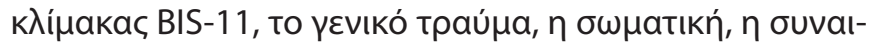

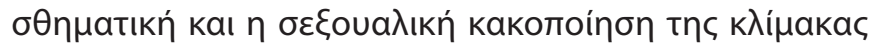

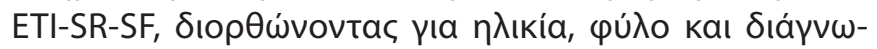

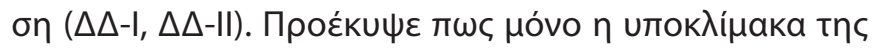

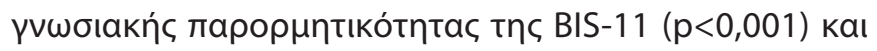

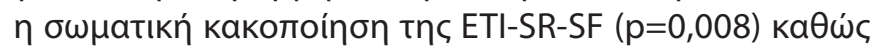

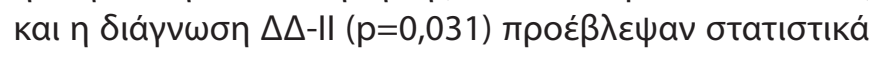

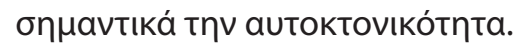

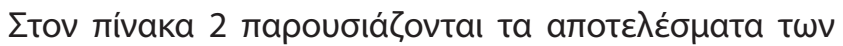

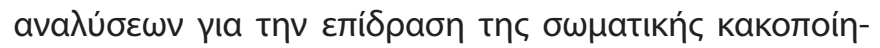

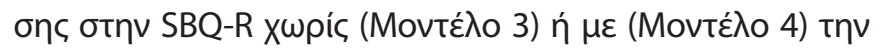

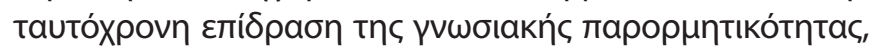

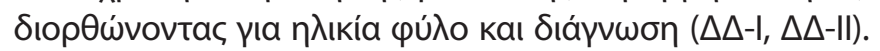

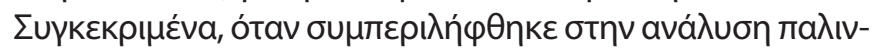

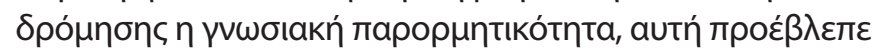

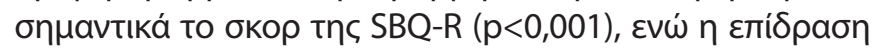

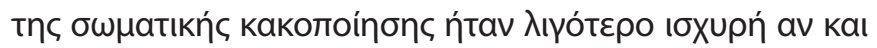

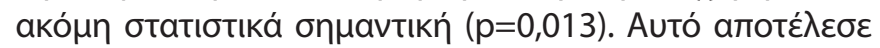

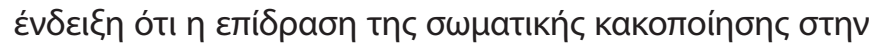

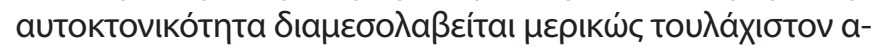

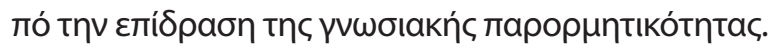

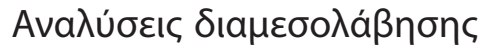

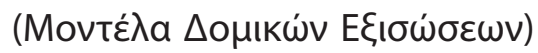

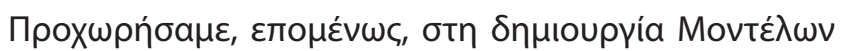

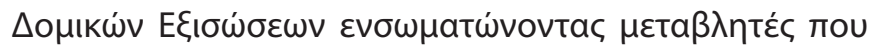

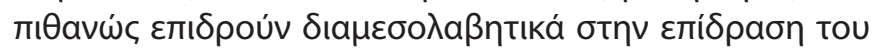

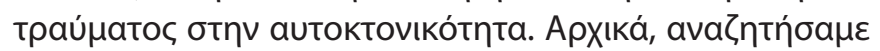

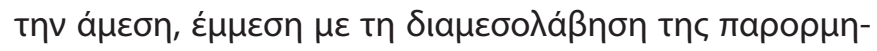

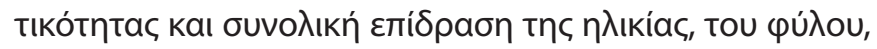

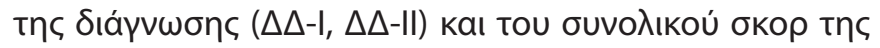

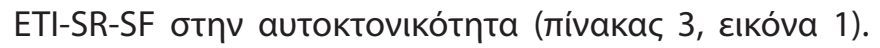




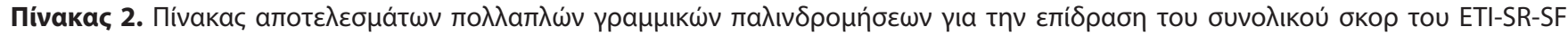

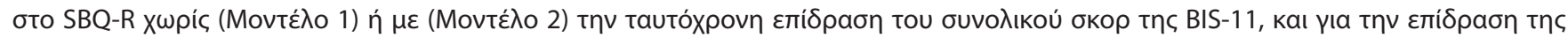

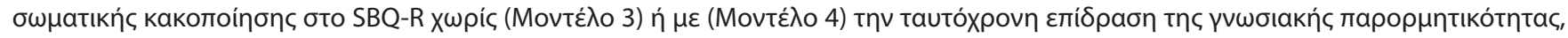

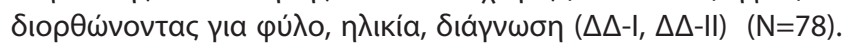

\begin{tabular}{|c|c|c|c|c|}
\hline 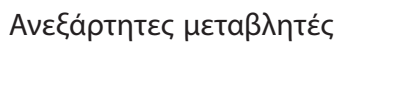 & beta & $\mathrm{p}$ & beta & $\mathrm{p}$ \\
\hline 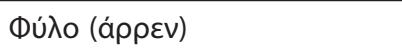 & 0,004 & 0,973 & $-0,004$ & 0,970 \\
\hline H入ıкі́a & 0,039 & 0,735 & $-0,045$ & 0,696 \\
\hline 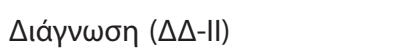 & 0,226 & 0,050 & 0,232 & 0,037 \\
\hline 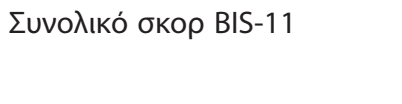 & \multicolumn{2}{|c|}{ 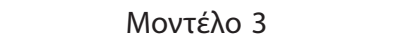 } & \multicolumn{2}{|c|}{ Movtéภo 4} \\
\hline 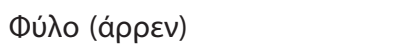 & $-0,027$ & 0,796 & $-0,022$ & 0,821 \\
\hline H入ıкía & 0,027 & 0,808 & $-0,014$ & 0,889 \\
\hline 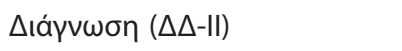 & 0,262 & 0,017 & 0,213 & 0,035 \\
\hline
\end{tabular}

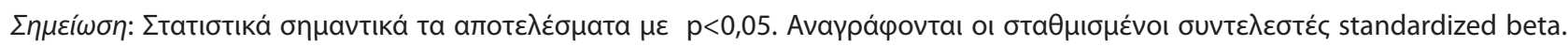

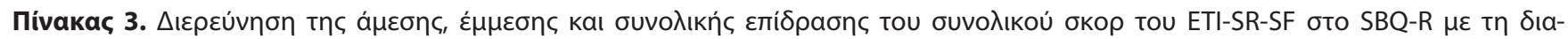

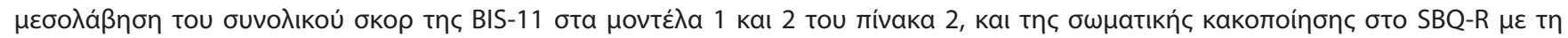

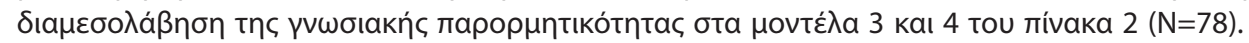

\begin{tabular}{|c|c|c|c|c|c|c|}
\hline & \multicolumn{2}{|c|}{ 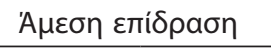 } & \multicolumn{2}{|c|}{ 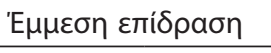 } & \multicolumn{2}{|c|}{ 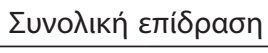 } \\
\hline & beta & $\mathrm{p}$ & beta & $\mathrm{p}$ & beta & $\mathrm{p}$ \\
\hline 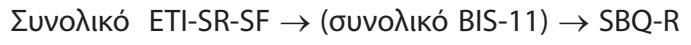 & 0,217 & 0,078 & 0,128 & 0,003 & 0,345 & 0,005 \\
\hline
\end{tabular}

tпTa) $\rightarrow$ SBQ-R

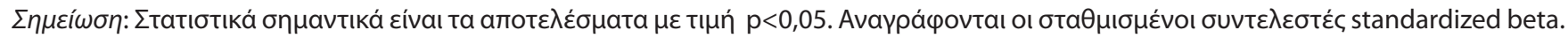

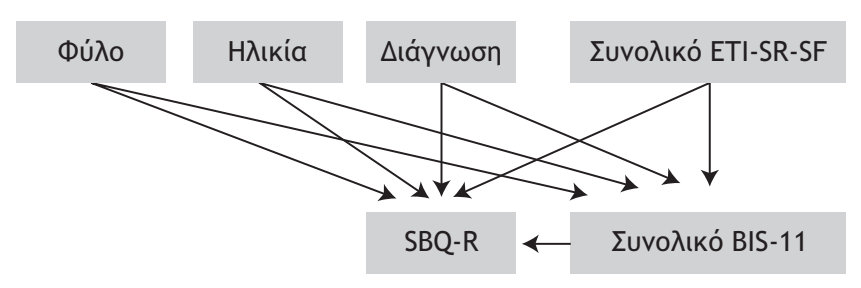

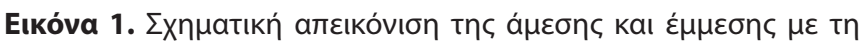

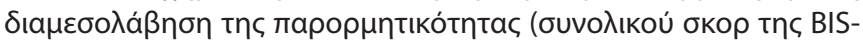

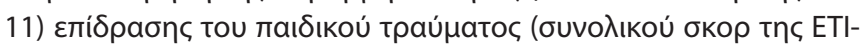

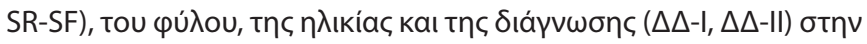

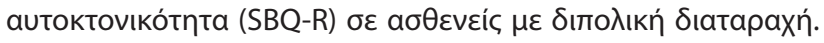

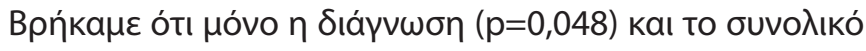

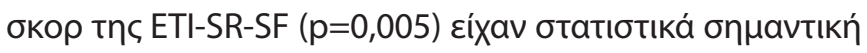

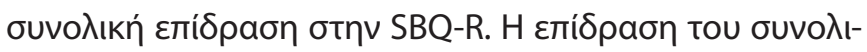

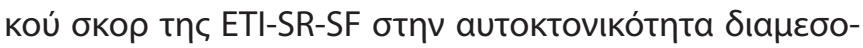

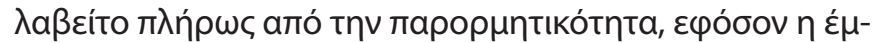

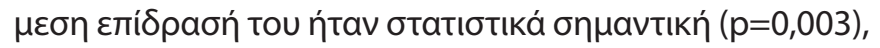

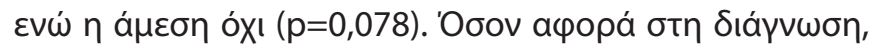

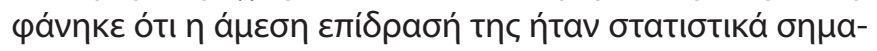

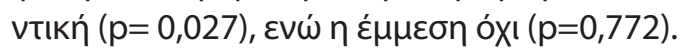

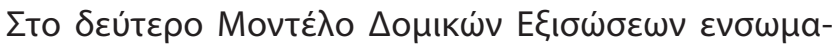

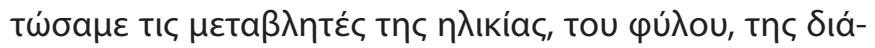

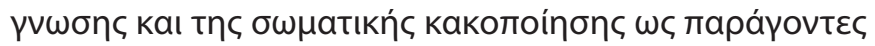

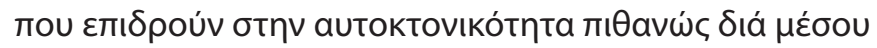

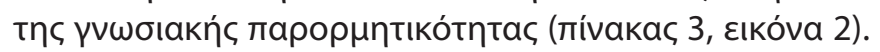

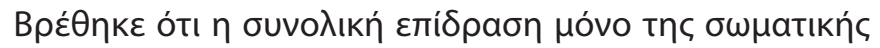

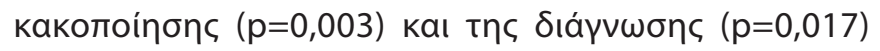

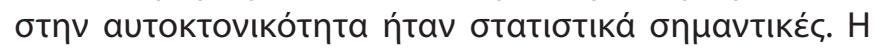

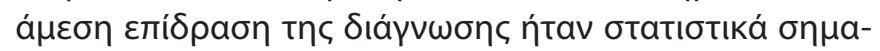

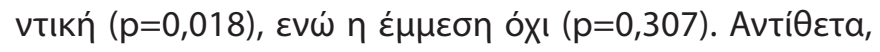

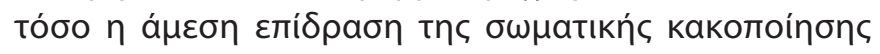




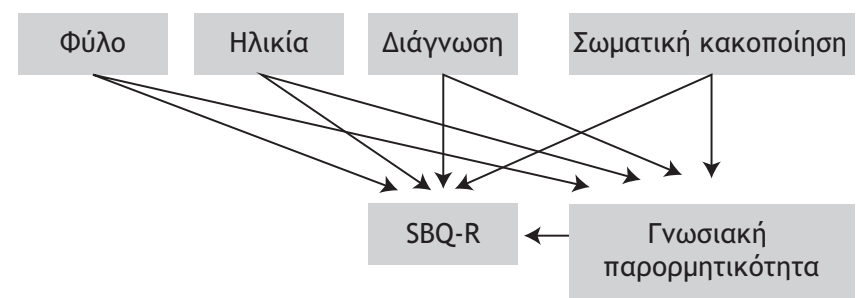

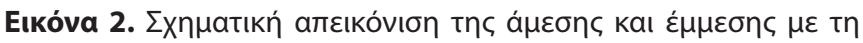

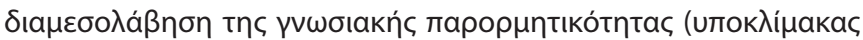

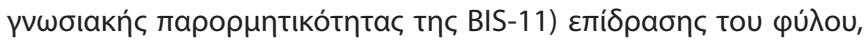

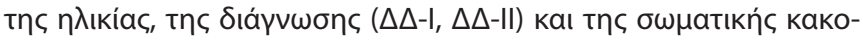

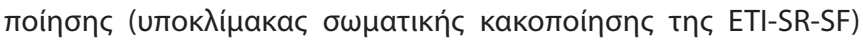

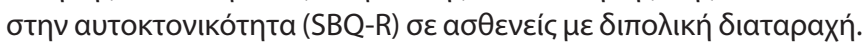

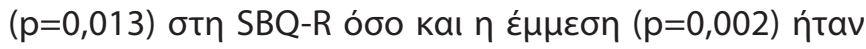

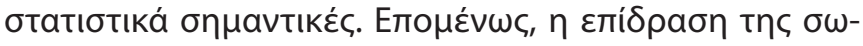

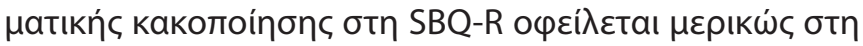

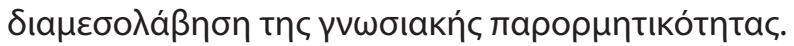

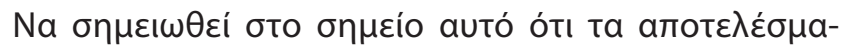

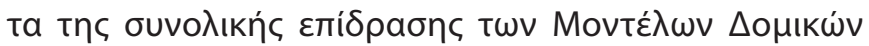

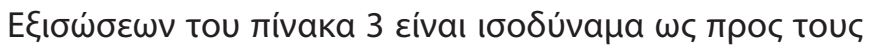

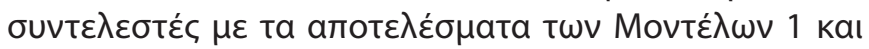

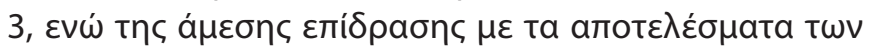

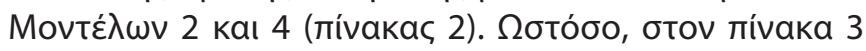

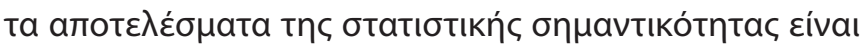

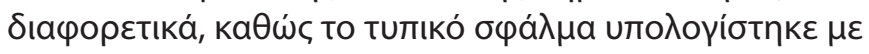

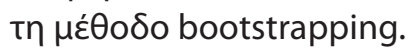

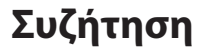

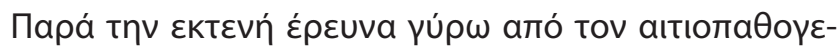

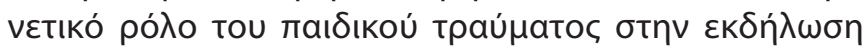

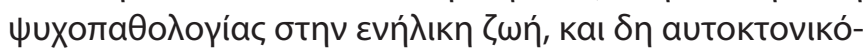

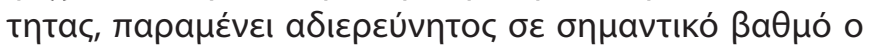

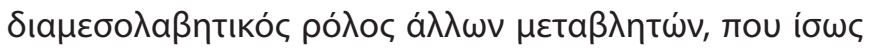

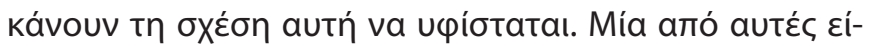

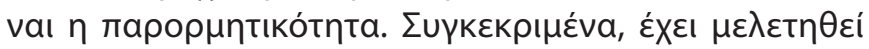

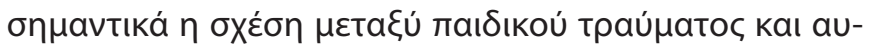

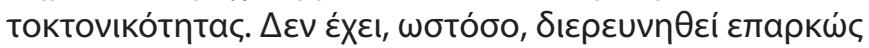

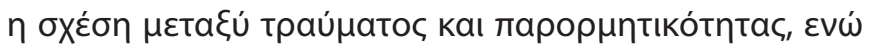

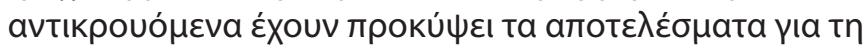

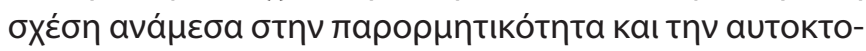
viкótnta.

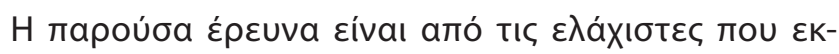

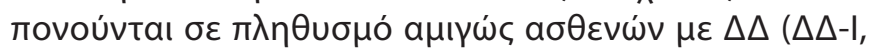

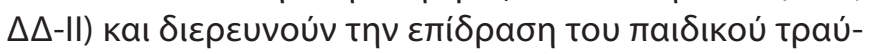

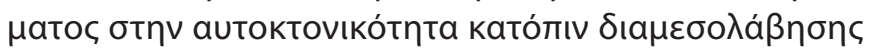

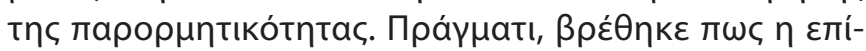

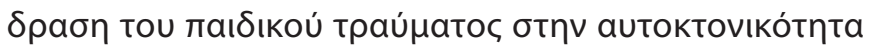

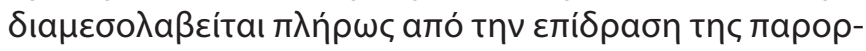

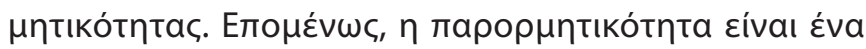

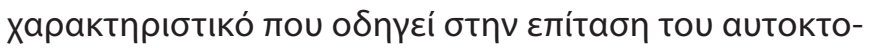

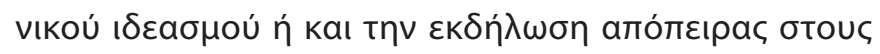

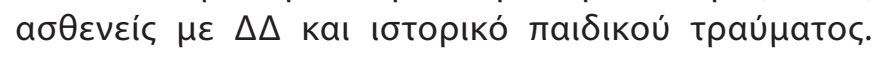

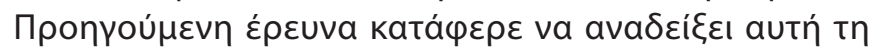

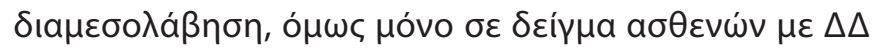

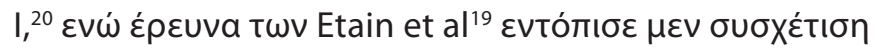

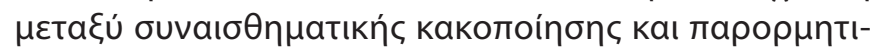

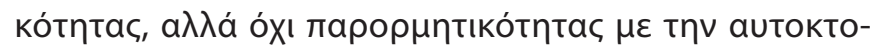

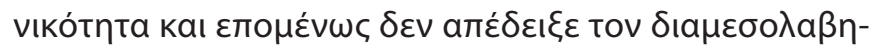

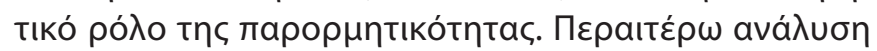

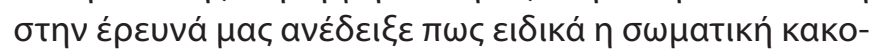

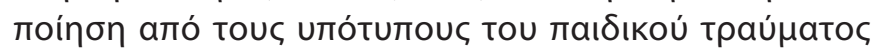

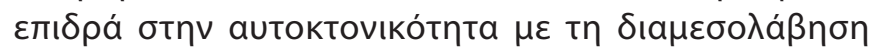

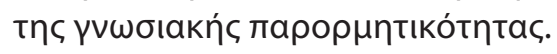

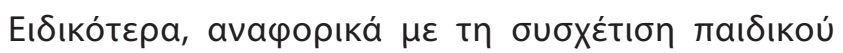

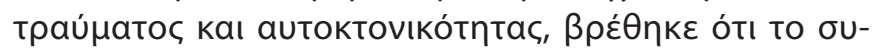

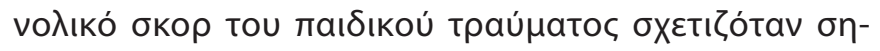

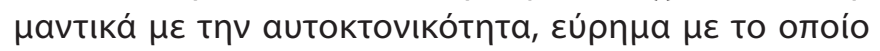

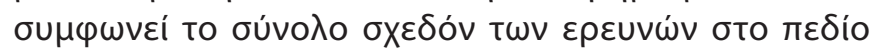

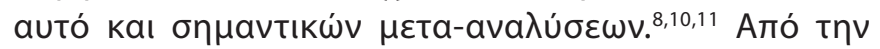

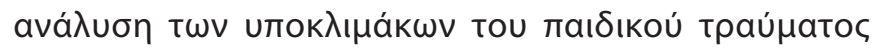

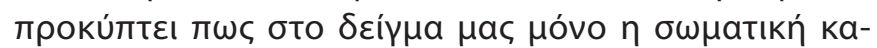

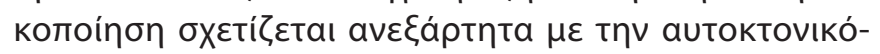

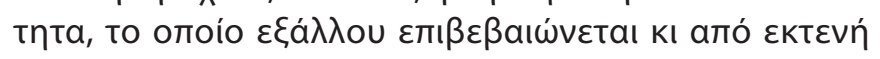

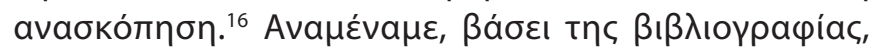

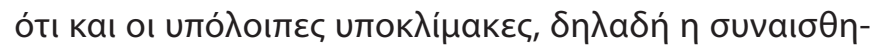

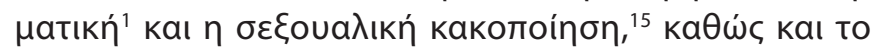

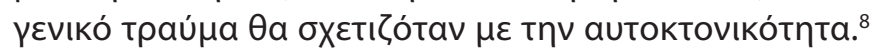

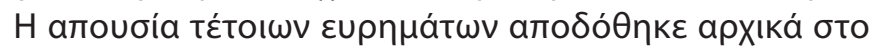

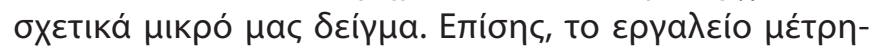

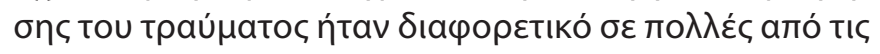

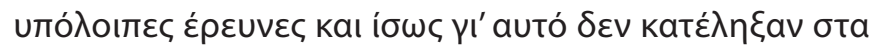

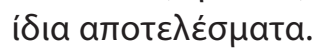

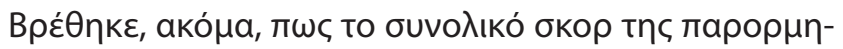

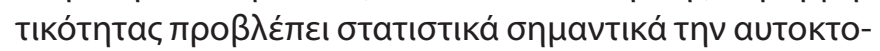

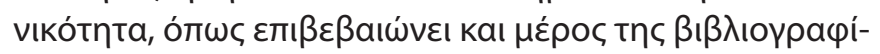

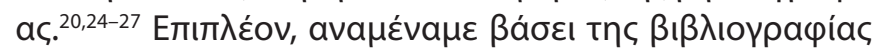

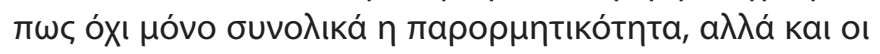

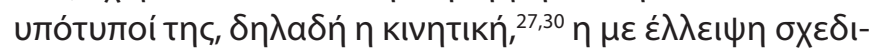

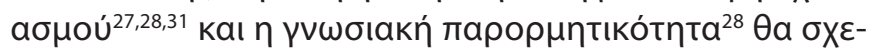

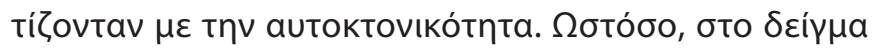

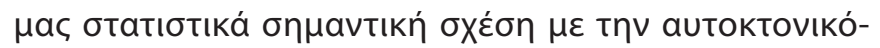

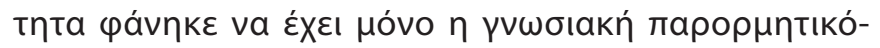

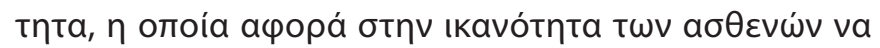

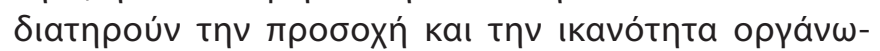

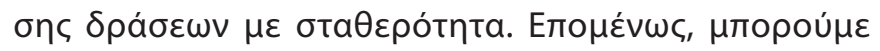

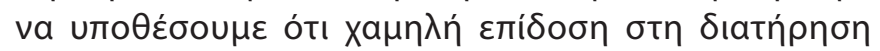

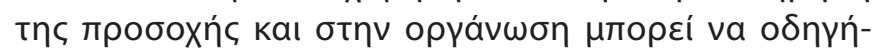

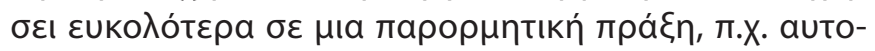

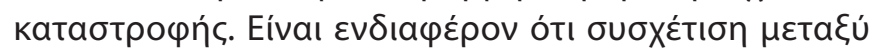

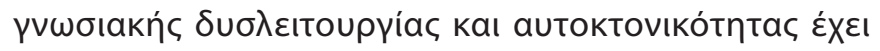

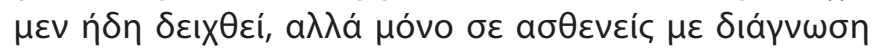

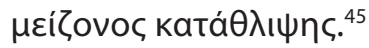




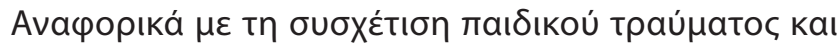

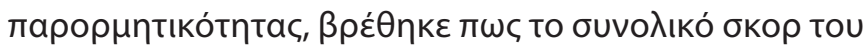

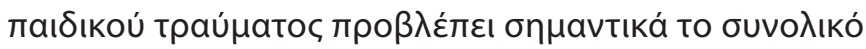

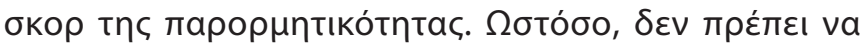

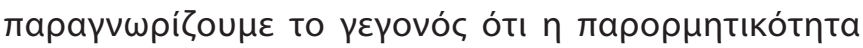

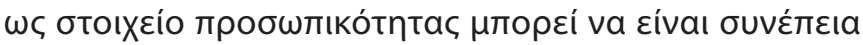

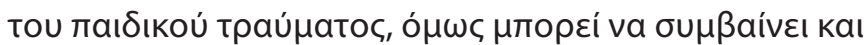

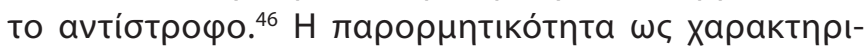

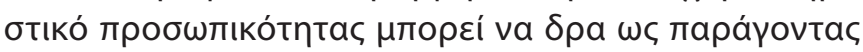

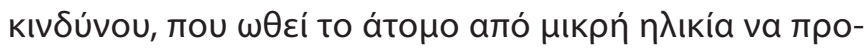

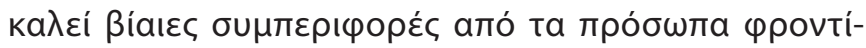

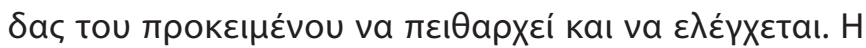

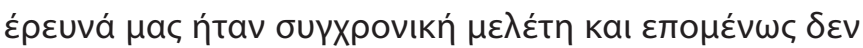

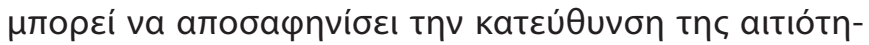

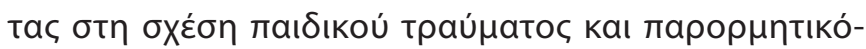

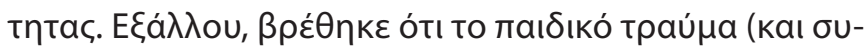

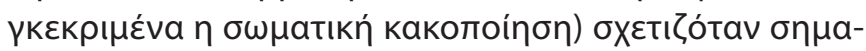

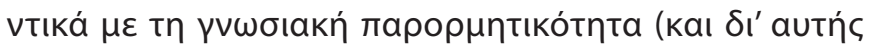

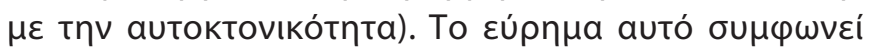

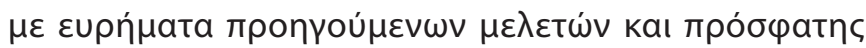
$\mu \varepsilon т a-a v a ́ \lambda u \sigma \eta \varsigma$ үıа тn $\delta$

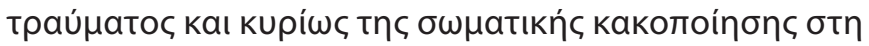

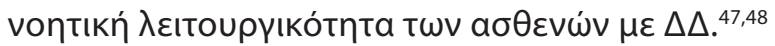

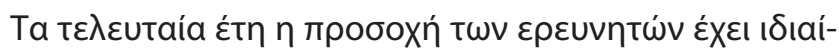

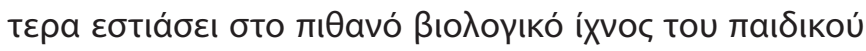

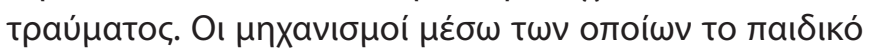

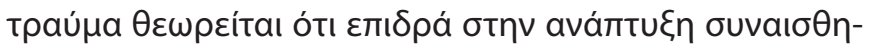

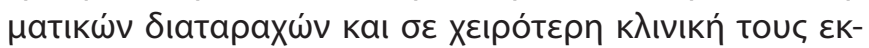

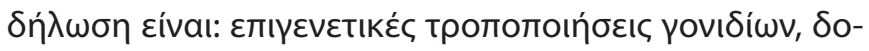

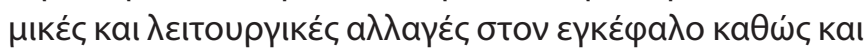

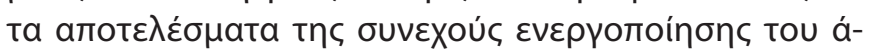

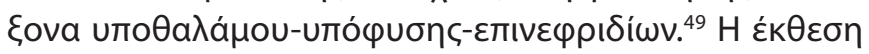

\section{BıBลtoypacpía}

1. Bremner JD, Bolus R, Mayer EA. Psychometric properties of the Early Trauma Inventory-Self Report. J Nerv Ment Dis 2007, 195:211-218, doi: 10.1097/01.nmd.0000243824.84651.6c

2. Bernstein DP, Fink L, Handelsman L, Foote J, Lovejoy M, Wenzel $K$ et al. Initial reliability and validity of a new retrospective measure of child abuse and neglect. Am J Psychiatry 1994, 151:1132-1136, doi: 10.1176/ajp.151.8.1132

3. World Health Organization and International Society for Prevention of Child Abuse and Neglect. Preventing child maltreatment: a guide to taking action and generating evidence. World Health Organization, Geneva, 2006

4. Carr CP, Martins CM, Stingel AM, Lemgruber VB, Juruena MF. The role of early life stress in adult psychiatric disorders: a systematic review according to childhood trauma subtypes. J Nerv Ment Dis 2013, 201:1007-1020, doi: 10.1097/NMD.0000000000000049

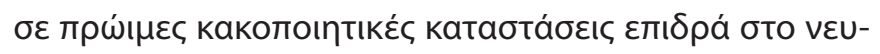

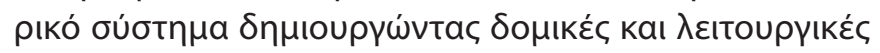

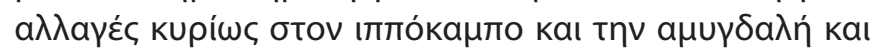

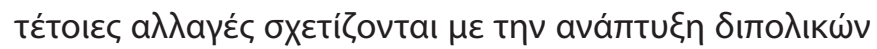

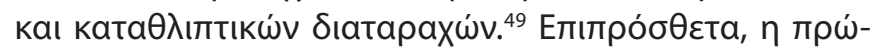

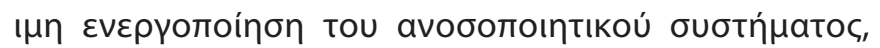

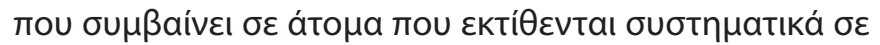

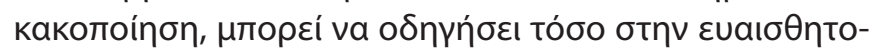

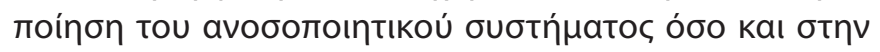

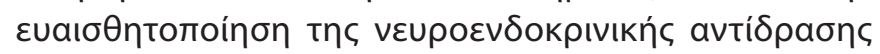
бто бтрє५. ${ }^{50}$

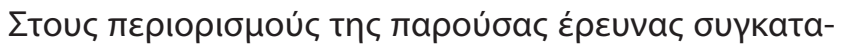

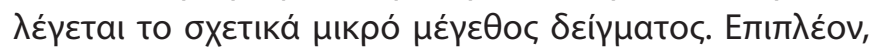

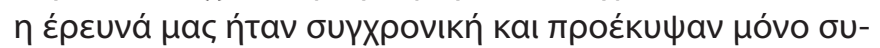

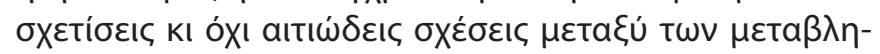
$\tau \omega ́ v$.

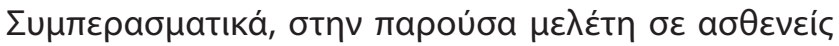

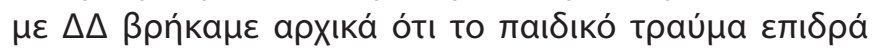

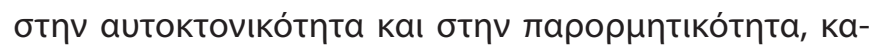

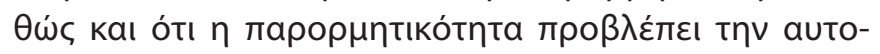

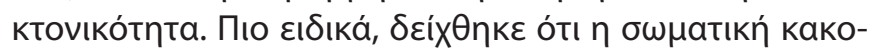

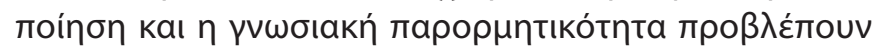

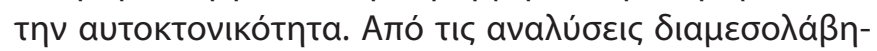

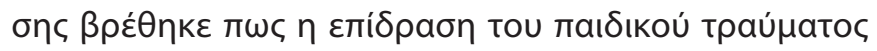

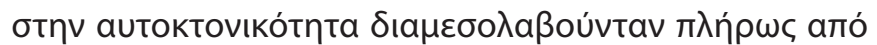

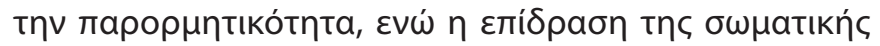

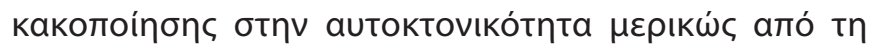

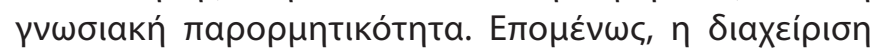

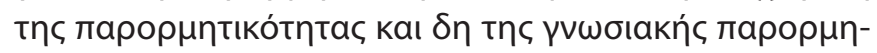

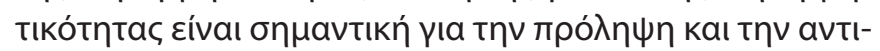

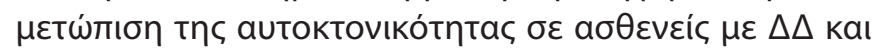

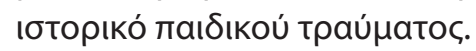

5. Sugaya L, Hasin DS, Olfson M, Lin KH, Grant BF, Blanco C. Child physical abuse and adult mental health: a national study. $J$ Trauma Stress 2012, 25:384-392, doi: 10.1002/jts.21719

6. Janiri D, Sani G, Danese E, Simonetti A, Ambrosi E, Angeletti G et al. Childhood traumatic experiences of patients with bipolar disorder type I and type II. J Affect Disord 2015, 175:92-97, doi: 10.1016/j. jad.2014.12.055

7. Marangoni C, Hernandez M, Faedda GL. The role of environmental exposures as risk factors for bipolar disorder: A systematic review of longitudinal studies. J Affect Disord 2016, 193:165-174, doi: 10.1016/j. jad.2015.12.055

8. Agnew-Blais J, Danese A. Childhood maltreatment and unfavourable clinical outcomes in bipolar disorder: a systematic review and meta-analysis. Lancet Psychiatry 2016, 3:342-349, doi: 10.1016/S22150366(15)00544-1

9. Aas M, Henry C, Andreassen OA, Bellivier F, Melle I, Etain B. The role of childhood trauma in bipolar disorders. Int J Bipolar Disord 2016, 4:2, doi: 10.1186/s40345-015-0042-0 
10. Etain B, Aas M, Andreassen OA, Lorentzen S, Dieset I, Gard S et al. Childhood trauma is associated with severe clinical characteristics of bipolar disorders. J Clin Psychiatry 2013, 74:991-998, doi: 10.4088/ JCP.13m08353

11. Larsson S, Aas M, Klungsoyr O, Agartz I, Mork E, Steen NE et al. Patterns of childhood adverse events are associated with clinical characteristics of bipolar disorder. BMC Psychiatry 2013, 13:97, doi: 10.1186/1471-244X-13-97

12. Costa Lda S, Alencar AP, Nascimento Neto PJ, dos Santos Mdo S, da Silva CG, Pinheiro Sde F et al. Risk factors for suicide in bipolar disorder: a systematic review. J Affect Disord 2015, 170:237-254, doi: 10.1016/j.jad.2014.09.003

13. Latalova K, Kamaradova D, Prasko J. Suicide in bipolar disorder: a review. Psychiatr Danub 2014, 26:108-114, PMID: 24909246

14. Mclntyre RS, Soczynska JK, Mancini D, Lam C, Woldeyohannes HO, Moon $\mathrm{S}$ et al. The relationship between childhood abuse and suicidality in adult bipolar disorder. Violence Vict 2008, 23:361-372, doi: 10.1891/0886-6708.23.3.361

15. Garno JL, Goldberg JF, Ramirez PM, Ritzler BA. Impact of childhood abuse on the clinical course of bipolar disorder. Br J Psychiatry 2005, 186:121-125, doi: 10.1192/bjp.186.2.121

16. Daruy-Filho L, Brietzke E, Lafer B, Grassi-Oliveira R. Childhood maltreatment and clinical outcomes of bipolar disorder. Acta Psychiatr Scand 2011, 124:427-434, doi: 10.1111/j.1600-0447.2011.01756.x

17. Swann AC, Moeller FG, Steinberg JL, Schneider L, Barratt ES, Dougherty DM. Manic symptoms and impulsivity during bipolar depressive episodes. Bipolar Disord 2007, 9:206-212, doi: 10.1111/ j.1399-5618.2007.00357.x

18. Liu RT. Childhood Maltreatment and Impulsivity: A Meta-Analysis and Recommendations for Future Study. J Abnorm Child Psychol 2019, 47:221-243, doi: 10.1007/s10802-018-0445-3

19. Etain B, Lajnef M, Henry C, Aubin V, Azorin JM, Bellivier F et al. Childhood trauma, dimensions of psychopathology and the clinical expression of bipolar disorders: A pathway analysis. J Psychiatr Res 2017, 95:37-45, doi: 10.1016/j.jpsychires.2017.07.013

20. Marwaha S, Briley PM, Perry A, Rankin P, DiFlorio A, Craddock N et al. Explaining why childhood abuse is a risk factor for poorer clinical course in bipolar disorder: a path analysis of 923 people with bipolar I disorder. Psychol Med 2019:1-9, doi: 10.1017/S0033291719002411

21. Richard-Lepouriel H, Kung AL, Hasler R, Bellivier F, Prada P, Gard S et al. Impulsivity and its association with childhood trauma experiences across bipolar disorder, attention deficit hyperactivity disorder and borderline personality disorder. J Affect Disord 2019, 244:33-41, doi: 10.1016/j.jad.2018.07.060

22. Gvion Y, Apter A. Aggression, impulsivity, and suicide behavior: a review of the literature. Arch Suicide Res 2011, 15:93-112, doi: 10.1080/ 13811118.2011.565265

23. Watkins HB, Meyer TD. Is there an empirical link between impulsivity and suicidality in bipolar disorders? A review of the current literature and the potential psychological implications of the relationship. Bipolar Disord 2013, 15:542-558, doi: 10.1111/bdi.12090

24. Mahon K, Burdick KE, Wu J, Ardekani BA, Szeszko PR. Relationship between suicidality and impulsivity in bipolar I disorder: a diffusion tensor imaging study. Bipolar Disord 2012, 14:80-89, doi: 10.1111/ j.1399-5618.2012.00984.x

25. Ostacher MJ, Lebeau RT, Perlis RH, Nierenberg AA, Lund HG, Moshier $\mathrm{SJ}$ et al. Cigarette smoking is associated with suicidality in bipolar disorder. Bipolar Disord 2009, 11:766-771, doi: 10.1111/j.1399-5618. 2009.00744.x

26. Sublette EM, Carballo JJ, Moreno C, Galfalvy HC, Brent DA, Birmaher $B$ et al. Substance use disorders and suicide attempts in bipolar subtypes. J Psychiatr Res 2009, 43:230-238, doi: 10.1016/j.jpsychires. 2008.05.001

27. Swann AC, Lijffijt M, Lane SD, Steinberg JL, Moeller FG. Increased trait-like impulsivity and course of illness in bipolar disorder. Bipolar Disord 2009, 11:280-288, doi: 10.1111/j.1399-5618.2009.00678.x

28. Ekinci O, Albayrak Y, Ekinci AE, Caykoylu A. Relationship of trait impulsivity with clinical presentation in euthymic bipolar disorder patients. Psychiatry Res 2011, 190:259-264, doi: 10.1016/j.psychres.2011.06.010

29. Etain B, Mathieu F, Liquet S, Raust A, Cochet B, Richard JR et al. Clinical features associated with trait-impulsiveness in euthymic bipolar disorder patients. J Affect Disord 2013, 144:240-247, doi: 10.1016/ j.jad.2012.07.005

30. Matsuo K, Nielsen N, Nicoletti MA, Hatch JP, Monkul ES, Watanabe $\mathrm{Y}$ et al. Anterior genu corpus callosum and impulsivity in suicidal patients with bipolar disorder. Neurosci Lett 2010, 469:75-80, doi: 10.1016/j.neulet.2009.11.047

31. Michaelis BH, Goldberg JF, Davis GP, Singer TM, Garno JL, Wenze SJ. Dimensions of impulsivity and aggression associated with suicide attempts among bipolar patients: a preliminary study. Suicide Life Threat Behav 2004, 34:172-176, doi: 10.1521/suli.34.2.172.32783

32. Oquendo MA, Waternaux C, Brodsky B, Parsons B, Haas GL, Malone $\mathrm{KM}$ et al. Suicidal behavior in bipolar mood disorder: clinical characteristics of attempters and nonattempters. J Affect Disord 2000, 59:107-117, doi: 10.1016/s0165-0327(99)00129-9

33. Parmentier C, Etain B, Yon L, Misson H, Mathieu F, Lajnef M et al. Clinical and dimensional characteristics of euthymic bipolar patients with or without suicidal behavior. Eur Psychiatry 2012, 27:570-576, doi: 10.1016/j.eurpsy.2011.05.005

34. Perroud N, Baud P, Mouthon D, Courtet P, Malafosse A. Impulsivity, aggression and suicidal behavior in unipolar and bipolar disorders. $J$ Affect Disord 2011, 134:112-118, doi: 10.1016/j.jad.2011.05.048

35. Swann AC, Dougherty DM, Pazzaglia PJ, Pham M, Steinberg JL, Moeller FG. Increased impulsivity associated with severity of suicide attempt history in patients with bipolar disorder. Am J Psychiatry 2005, 162:1680-1687, doi: 10.1176/appi.ajp.162.9.1680

36. Gilbert AM, Garno JL, Braga RJ, Shaya Y, Goldberg TE, Malhotra AK et al. Clinical and cognitive correlates of suicide attempts in bipolar disorder: is suicide predictable? J Clin Psychiatry 2011, 72:1027-1033, doi: 10.4088/JCP.10m06410

37. Sheehan DV, Lecrubier $Y$, Sheehan $\mathrm{KH}$, Amorim $\mathrm{P}$, Janavs J, Weiller E et al. The Mini-International Neuropsychiatric Interview (M.I.N.I.): the development and validation of a structured diagnostic psychiatric interview for DSM-IV and ICD-10. J Clin Psychiatry 1998, 59 (Suppl 20):22-33, PMID: 9881538

38. Hamilton M. A rating scale for depression. J Neurol Neurosurg Psychiatry 1960, 23:56-62, doi: 10.1136/jnnp.23.1.56

39. Young RC, Biggs JT, Ziegler VE, Meyer DA. A rating scale for mania: reliability, validity and sensitivity. Br J Psychiatry 1978, 133:429-435, doi: 10.1192/bjp.133.5.429

40. Antonopoulou Z, Konstantakopoulos G, Tauzinieri-Coccosis M, Sinodinou C. Rates of childhood trauma in a sample of university students in Greece: The Greek version of the Early Trauma Inventory-Self Report. Psychiatriki 2017, 28:19-27, doi: 10.22365/jpsych.2017.281.19 
41. Osman A, Bagge CL, Gutierrez PM, Konick LC, Kopper BA, Barrios FX. Suicidal Behaviours Questionnaire-Revised (SBQ-R): Validation with clinical and nonclinical samples. Assessment 2001, 8:443-454, doi: 10.1177/107319110100800409

42. Patton JH, Stanford MS, Barratt ES. Factor structure of the Barratt impulsiveness scale. J Clin Psychol 1995, 51:768-774, doi: 10.1002/ 1097-4679(199511)51:6<768:aid-jclp2270510607>3.0.co;2-1

43. Stanford MS, Mathias CW, Dougherty DM, Lake SL, Anderson NE, Patton JH. Fifty years of the Barratt Impulsiveness Scale: An update and review. Pers Individ Dif 2009, 47:385-395, doi: https://doi.org/ 10.1016/j.paid.2009.04.008

44. Giotakos O, Markianos M, Vaidakis N, Christodoulou GN. Aggression, impulsivity, plasma sex hormones, and biogenic amine turnover in a forensic population of rapists. J Sex Marital Ther 2003, 29:215-225, doi: 10.1080/00926230390155113

45. Pu S, Setoyama S, Noda T. Association between cognitive deficits and suicidal ideation in patients with major depressive disorder. Sci Rep 2017, 7:11637, doi: 10.1038/s41598-017-12142-8
46. Braquehais MD, Oquendo MA, Baca-Garcia E, Sher L. Is impulsivity a link between childhood abuse and suicide? Compr Psychiatry 2010, 51:121-129, doi: 10.1016/j.comppsych.2009.05.003

47. Jimenez E, Sole B, Arias B, Mitjans M, Varo C, Reinares M et al. Impact of childhood trauma on cognitive profile in bipolar disorder. Bipolar Disord 2017, 19:363-374, doi: 10.1111/bdi.12514

48. Dauvermann MR, Donohoe G. The role of childhood trauma in cognitive performance in schizophrenia and bipolar disorder - A systematic review. Schizophr Res Cogn 2019, 16:1-11, doi: 10.1016/j. scog.2018.11.001

49. Jaworska-Andryszewska P, Rybakowski JK. Childhood trauma in mood disorders: Neurobiological mechanisms and implications for treatment. Pharmacol Rep 2019, 71:112-120, doi: 10.1016/j. pharep.2018.10.004

50. Danese A, Lewis SJ. Psychoneuroimmunology of Early-Life Stress: The Hidden Wounds of Childhood Trauma? Neuropsychopharmacology 2017, 42:99-114, doi: 10.1038/npp.2016.198 


\title{
Childhood trauma and suicidality in bipolar disorder: The mediatory role of impulsivity
}

\author{
Sofia Dramilaraki, Anastasia Antoniou, Evgenia Porichi, Vassiliki Efstathiou, loannis \\ Michopoulos, Rossetos Gournellis, Athanassios Douzenis, Panagiotis Ferentinos
}

Second Department of Psychiatry, National and Kapodistrian University of Athens, Attikon University Hospital, Athens, Greece

ARTICLE HISTORY: Received 18 November 2020 / Revised 2 January 2021 / Published Online 28 May 2021

\begin{abstract}
Childhood trauma (CT) is correlated with suicidality among patients with bipolar disorder (BD). However, it has not been adequately investigated if a third factor, for instance impulsivity, mediates the effect of CT on suicidality in BD. This study aimed to explore potential mediatory effects of impulsivity in the pathway from CT to suicidality in BD. CT was assessed with the Early Trauma Inventory Self Report-Short Form (ETI-SR-SF), impulsivity with the Barratt Impulsivity Scale-11 (BIS-11) while lifetime suicidality was investigated with the Suicidal Behaviors Questionnaire-Revised (SBQ-R). The effect of childhood trauma on suicidality and impulsivity as well as the effect of impulsivity on suicidality were examined with multiple linear regressions, including gender, age and diagnosis (BD-I, BD-II) as covariates. Structural equation models were built and path analyses were performed (with AMOS 25 software and using bootstrapping in 1000 samples) for the examination of the mediatory role of BIS-11 and its subtypes in the effect of childhood trauma and its subtypes on suicidality. We included 78 BD euthymic patients (60.3\% female, $67.9 \%$ BD-I). ETI-SR-SF significantly predicted SBQ-R $(p=0.004)$ and BIS-11 ( $p<0.001)$, while BIS-11 significantly predicted SBQ-R ( $p=0.001)$. In a model including ETI-SR-SF and BIS dimensions, only ETISR-SF physical abuse ( $p=0.012)$ and BIS attentional impulsiveness $(p<0.001)$ subscales significantly predicted SBQ-R. In structural equation models, the indirect effect of childhood trauma on suicidality via impulsivity was significant $(p=0.003)$ while the direct effect of childhood trauma on suicidality was non-significant (complete mediation of the effect of childhood trauma on suicidality via impulsivity). In specific, both the indirect effect of physical abuse on suicidality via attentional impulsivity $(p=0.002)$ and the direct effect of physical abuse on suicidality $(p=0.013)$ were statistically significant (partial mediation of the effect of physical abuse on suicidality via attentional impulsivity). Childhood trauma predicts suicidality and impulsivity, while impulsivity predicts suicidality. More specifically, physical abuse and attentional impulsivity predict suicidality. The mediatory role of impulsivity completely explained the effect of childhood trauma on suicidality. Moreover, attentional impulsivity partially mediated the effect of physical abuse on suicidality. Therefore, management of impulsivity and especially of attentional impulsivity is crucial for the prevention and management of suicidality among BD patients with a history of childhood trauma.
\end{abstract}

KEYWORDS: Childhood trauma, impulsivity, suicidality, bipolar disorder. 